\title{
The Effects of Cross-modal Collaboration on the Stages of Information Seeking
}

\author{
Dena Al-Thani, Tony Stockman, Anastasios Tombros \\ School of Electronic Engineering and Computer Science \\ Queen Mary University of London \\ Mile End road, London, E1 4NA, UK \\ \{d.al-thani, tony.stockman, a.tombros\}@qmul.ac.uk
}

\begin{abstract}
Previous studies of users with visual impairments access to the web have focused on human-web interaction. This study explores the under investigated area of cross-modal collaborative information seeking (CCIS), that is, the challenges and opportunities that exist in supporting visually impaired (VI) users to take an effective part in collaborative web search tasks with sighted peers. We conducted an observational study to investigate the process with fourteen pairs of VI and sighted users in colocated and distributed settings. The study examined the effects of cross-modal collaborative interaction on the stages of the individual Information Seeking (IS) process. The findings showed that the different stages of the process were performed individually most of the time; however it was observed that some collaboration took place in the results exploration and management stages. The accessibility challenges faced by VI users affected their individual and collaborative interaction and also enforced certain points of collaboration. The paper concludes with some recommendations towards improving the accessibility of cross-modal collaborative search.
\end{abstract}

\section{Categories and Subject Descriptors}

H.5.3 [Group and Organization Interfaces]: Collaborative computing; K.4.2 [Social Issues]: Assistive technologies for persons with disabilities

\section{General Terms}

Human Factors, Experimentation, Design

\section{Keywords}

Collaborative information seeking; cross-modal interaction, information seeking process; accessibility; web search

\section{INTRODUCTION}

In the context of Information Seeking (IS), observational studies reveal that group members often collaborate when searching for information, even if they were not explicitly asked to do so [15][12] The activity that involves a group of people searching in a common information seeking task is called Collaborative

Permission to make digital or hard copies of all or part of this work for personal or classroom use is granted without fee provided that copies are not made or distributed for profit or commercial advantage and that copies bear this notice and the full citation on the first page. Copyrights for components of this work owned by others than ACM must be honored. Abstracting with credit is permitted. To copy otherwise, or republish, to post on servers or to redistribute to lists, requires prior specific permission and/or a fee. Request permissions from Permissions@ acm.org.

Interacción '15, September 07-09, 2015, Vilanova i la Geltrú, Spain (C) 2015 ACM. ISBN 978-1-4503-3463-1/15/09..\$15.00 DOI: http://dx.doi.org/10.1145/2829875.2829925
Information Seeking (CIS). Over the past few years, research in this area has been gaining much interest. This attention on multiuser IS has always assumed all group members are using visual displays. This focus on the visual modality limits the relevance of previous research to users employing other interaction modes for accessing and managing retrieved results. This paper presents the results of an exploratory study conducted to investigate the effect of the presence of two different modalities on the process of CIS. We term the process under investigation cross-modal, collaborative Information Seeking (CCIS). The purpose of this study is to better understand the CCIS process and its effects on stages of the individual IS process presented by Marchionini and White [14].

To date, very few studies [24][26] have examined the process of CIS or attempt to draw a framework and models that describe its processes, therefor in our paper we take the Marchionini and White [14] model of individual information seeking and map its processes to the individual and collaborative IS activities performed. The way their model introduces the IS activity as a process that includes discreet stages will help to inform our understanding of how users employing different modalities go about performing each stage of the IS process both individually and collaboratively. The project is motivated by the observation that many activities in both educational and work settings involve teamwork, and that internet searching often forms an important component of such activities. Specifically, we wish to understand what barriers may exist to visually impaired searchers taking part in CCIS, and what approaches are currently employed by CCIS participants to overcome or work around these barriers.

The paper starts with a brief overview of related work on the accessibility of the single user IS process and a summary of the current research on CIS. We then present our motivation and research questions, before describing the details of the study and results obtained. The effects of cross-modality on the awareness and division of labour in CIS involving VI and sighted collaborators have been reported elsewhere [1], where the patterns of behavior to achieve awareness and strategies to divide labor are described. This paper focuses on examining the effects of crossmodality on the structure of the IS process and identifying the stages in which collaboration occurred and the reasons for it taking place. This paper concludes by discussing the implications of the results to inform the design of a tool that supports CCIS activities.

\section{RELATED BACKGROUND}

\subsection{Accessible Information Seeking}

Despite the fact that issues surrounding web accessibility have attracted increased attention in research and in the commercial world [3][7], the area of accessible IS is rarely examined. The 
sequential nature of screen-readers imposes many challenges on visually impaired (VI) web users. These challenges range from the lack of context to overload of short-term memory. Studies have highlighted these challenges and proposed a set of guidelines to be considered when designing an accessible search engine [2][5]. While these studies focused on the usability aspects of the problem, a recent study by Sahib et al. [22] examined the challenges that this problem imposes on the different stages of the IS process and hence on the behaviour of the VI information seeker.

In the comparative analysis of Sahib et al. [22] an observational user study was conducted with 15 VI and 15 sighted participants. The participants were asked to perform a complex task which required a high level of cognitive effort (i.e., detailed planning of a vacation). The results of the comparative study identified major differences between the IS behaviour of VI and sighted participants. These differences were particularly apparent in the query formulation and results exploration stages. One of the main barriers highlighted was the inaccessibility to screen-reader users of query-level support features provided by search engines at the query formulation phase. Also, In the search exploration stage the number of results viewed by VI participants (mean of 4.27 (SD= 2.15) web search results viewed) was considerably lower than the number of results viewed by sighted participants (mean of 13.40 $(\mathrm{SD}=7.39))$. These findings led to the development and evaluation of a search interface [23] that aim to tackle the issues identified in their study with special attention to the results exploration and managements stages of the single user IS process.

\subsection{Collaborative Information Seeking}

Collaborative information seeking is defined as the activity performed by a group of people with a shared information need or 'goal' [15]. A survey by Morris [15] is referred to as one of the earliest studies that encouraged increased attention in this area. Her survey, which she revisited lately [16] did not attempt to examine users' behavior, yet it provided a wealth of data regarding the prevalence of collaborative web search and the tasks motivation and tools involved.

Despite the extensive research in this field in the past few years, there is no consensus over a single model or framework that describes the CIS process. Though there have been a number of research attempts to develop models either to describe the CIS environment [24] or to classify the systems supporting it [6]. Shah [24] proposed a layered model of information seeking. The model contains four layers which are information, tools, users and results. The information layer refers to the different resources and formats of information contained in the entire search space. The tools basically refer to the search engines and the functionality they provide. The user layer includes the users, their profiles and any mechanisms available for personalization. The final layer is the results, ultimately the product of the search process, including all relevant information, users' comments and metadata.

Studies [8][26] examined the applicability of Kuhthau's [11] process of individual information seeking in the context of a group. Hyldegard [8] observed a group of 10 students over 14 weeks when performing information seeking activities and Shah and Gonzalez-Ibanez [26] conducted a laboratory study involving 42 pairs performing a general exploratory search task. Both concluded that though there are evident similarities in the general stages of the process between individual and collaborative behaviours in information seeking, there were also important differences. The differences are related to the contextual aspects associated with social factors. The results of the studies were similar and both concluded that Kuhlthau's process did not completely address the social dimension of CIS.

Golovchinsky et al. [6] proposed a taxonomy of CIS collaboration in which they introduced four dimensions of collaboration. The focus of this taxonomy is on technical models of collaboration rather than social models, they proposed four different dimensions of collaboration, which are intent, depth of mediation, concurrency and location. (1) Intent: Explicit vs. Implicit: When implicit collaboration is supported, the search engine uses data from previous anonymous users with similar information needs or similar behaviour to offer recommendations to users. Recommender systems thus support implicit collaboration. In contrast, in systems that support explicit collaboration, users explicitly work together in the query formation and results exploration stages. Microsoft's SearchTogether [17] system is an example of an application that supports explicit collaboration. (2) Depth of Mediation: This dimension refers to the level in which the mediation of information seeking is applied in a system. Pickens et al [20] introduced Cerchiamo, in which CIS is mediated at the algorithmic level. The Cerchiamo collaborative search engine divides the labour between two collaborators. One collaborator is the "preceptor" who investigates new fields of information, while the other collaborator is the "surveyor," who looks at and explores each new field in detail. (3) Concurrency: Synchronous vs. Asynchronous: Concurrency, which does not actually relate to time, means that the system should allow the actions of a user to be conveyed in some way to other team members. In other words, systems should support awareness between collaborative users within a group engaged in different information seeking activities. (4) Location: Co-located vs. Distributed: Distributed collaboration may require additional communication channels such as instant messaging, offline messaging services and voice chat.

\section{RESEARCH QUESTIONS AND MOTIVATION}

The increased interest in CIS reflects the fact that it is a more frequent activity in our daily lives. However, there has not previously been any attempt to consider the way CIS activities may be different when collaborators use different interface modalities, which is the focus of the current study. The questions we wished to examine are as follows:

Q1: What stages of the information seeking process are done collaboratively and how?

Studies have revealed that though IS stages are typically done individually. Nevertheless collaborators may choose to work together at many points in the process [26][27]. These studies also stressed that collaborative activities are generally ill structured [13]. Hence, very few efforts have been made to define a model of CIS activity [8][26]. In addressing this question, we aim to explore how often collaboration occurs at each stage, how much collaboration takes place and what techniques are used to support it? Furthermore, we wish to examine the effects of cross modality on group performance and techniques employed to address issues arising from the use of different interaction modes. For example, previous research on VI users IS behaviour has highlighted that most challenges are encountered during the results exploration phase [22]. These difficulties arise because examining large search result sets using a screen reader can be a lengthy process, due to 
the sequential nature of speech and other limitations relating to the navigation of complex information with a speech-based screen reader [19][28].

Q2: What are the strategies and techniques employed to manage search results?

This question also explores the management of search results in the presence of a common goal between group members who use different access modalities. It seeks to identify approaches and techniques used to organize, exchange and manage search results.

\section{OBSERVATIONAL STUDY}

We observed 14 pairs of users, each pair comprising one sighted and one VI partner, performing two CCIS tasks. For one of the tasks the partners were co-located, while in the other they were located separately. Task order and location were balanced to counter learning effects.

\subsection{Participants}

We recruited 28 participants, 14 sighted and 14 VI, via mailing lists; table 1 contains their demographic data and the technologies they used. Three VI users employed headphones for speech output, while the other five used speakers. All the VI users used the speech-only version of the Jaws ${ }^{1}$ screen-reader. Two pairs were colleagues for more than two years. None of the other pairs had worked together on a regular basis.

\begin{tabular}{|c|c|c|}
\hline & $\begin{array}{l}\text { Visually Impaired } \\
\text { Participants }\end{array}$ & $\begin{array}{c}\text { Sighted } \\
\text { Participants }\end{array}$ \\
\hline Age & $\begin{array}{l}2(21-29), 4(30-39), \\
3(40-49), 5(50-59)\end{array}$ & $\begin{array}{c}2(18-20), 3(21-29), \\
3(40-49), 5(30- \\
39), 1(50-59)\end{array}$ \\
\hline Gender & 8 Male,6 Female & 8 Male, 6 Female \\
\hline $\begin{array}{l}\text { Browser } \\
\text { Used } \\
\text { (Multiple } \\
\text { Answers) }\end{array}$ & $\begin{array}{c}12 \text { IE, } 8 \text { Safari, } 5 \\
\text { Firefox }\end{array}$ & $\begin{array}{c}6 \text { IE, } 4 \text { Firefox } \\
3 \text { Safari, } 1 \text { Chrome }\end{array}$ \\
\hline $\begin{array}{l}\text { Frequency } \\
\text { of CIS } \\
\text { Activity }\end{array}$ & $\begin{array}{l}3 \text { Daily, } 2 \text { Once a } \\
\text { week, } 5 \text { once a month } \\
1 \text { Once in the past six } \\
\text { months, } 3 \text { Never }\end{array}$ & $\begin{array}{c}2 \text { Weekly, } 3 \text { once a } \\
\text { month, } 6 \text { Once in } \\
\text { the past six months, } \\
3 \text { Never }\end{array}$ \\
\hline
\end{tabular}

Table 1. Demographic and technology information about participants

\subsection{Tasks}

Previous CIS research has identified that simple information lookups and fact finding tasks do not benefit from CIS activity, while multi-facetted and exploratory search tasks are likely to be more appropriate for use in CIS investigations [13][17]. Therefore, participants were asked to collect relevant information for two exploratory tasks that were designed to be realistic work and leisure tasks respectively. The task used in the co-located session was to organize a business trip to the United States (US) while the task in the distributed session was to organize a holiday trip to Australia. They were given dates of engagements in different cities and times when leisure or work activities needed to be

\footnotetext{
${ }^{1}$ http://www.freedomscientific.com/
}

identified. Participants were asked to organize the travel, accommodation and activities in these cities. In advance of each study we made sure that participants had not visited the cities before. The complexity of the two tasks was counterbalanced to make them approximately equal in their level of difficulty. They were balanced for subtasks and amount of information retrieved.

\subsection{Sessions}

Both the co-located and distributed sessions took place at the VI participants' workplace as the intention was to observe the participants in real world settings. For the same reason they were asked to use their own PCs and the web browser and search engines they normally used. In the distributed sessions, participants were seated in remote locations and told that they could use one or more of the following methods to communicate: email, instant messaging, shared documents, or any tool they found suitable. While in the co-located setting, participants were seated in the same room and asked to communicate verbally, though they were free to use additional methods if desired.

During the first session, participants were briefed about the purpose of the study and asked to fill a pre-study questionnaire which collected their demographic data, information about the technologies they use for this type of task and their level of experience with web searching. In each session, they were provided with a brief document giving information about the trip they were required to organise, including dates when they needed to be in different places and details of the types of activities they were required to book.

Following that, participants were asked to perform the tasks and about 35 minutes into their work the principle researcher asked them to stop. We intentionally did not inform them in advance about the amount of time they have to perform the task as we were not interested in examining the influence of time pressure in this study. We concluded each session with a brief semi-structured interview to discuss the participants' experience of the task.

All sessions were videotaped, having obtained the approval of the participants. During the tasks, the screens of both participants were captured using screen recording software. The VI participants' screens were captured using a video camera, as we noticed in a pilot of the experiment that screen recording software sometimes reduced the responsiveness of screen-readers. Additionally the principle researcher made notes of observations during the sessions.

\subsection{Data Collection}

The main source of data was the video recordings of the interactions between partners and their interactions with the search engines and the post-study interviews. After transcribing the videos, we used the Open and Axial coding phases of grounded theory [4]. Open coding is the process of generating initial concepts from the data while axial coding is when the data is put together to establish connections between the different concepts and categories. The selective coding process includes the formalisation of the data into theoretical frameworks. However, for this study, we stopped our data analysis after open and axial coding as we wanted only to explore the behaviour of the collaborating searchers, as opposed to developing a new theory.

The coding scheme captured indicators of each IS process stage. In relation to the interactions between partners, the coding scheme captured instances of collaborative IS activities and the reasons for 
these taking place. Semi-structured interviews were conducted individually with each participant to complement the data collected during the study. On the quantitative data, we carried out statistical testing at $\mathrm{p}<0.05$ with a two-tailed unpaired t-test.

\section{ANALYSIS}

\subsection{Stages of the Collaborative Process}

In general, the process started with a stage in which the pair divided the tasks to be performed. At this stage, usually one of the participants took the lead and assigned tasks to themselves and to their partner. During this process, the other partner might either agree on the task s/he is being given or suggest another task. For instance, visually impaired experienced web users sometimes anticipated that certain tasks were likely to require a longer time for them to complete, therefore they sometimes suggested they performed other tasks. Seven VI participants in the co-located setting and 10 VI participants in the distributed setting preferred searching for a tourism site to booking a hotel room, because the latter task involved filling an online form. In the interviews, VI participants explained that from previous experience of filling web forms they knew that this process can sometimes be lengthy or not feasible due to the presence of inaccessible form elements.

In the co-located sessions, an iterative process was observed. This process mainly involved three stages. In the first stage the pair spent from 2 to 5 minutes looking into and discussing the task. The discussion at this stage mainly related to an initial division of labour. At this stage the task was divided into smaller sub-tasks. However in the majority of cases, partners only decided on who would do each of the first sub-tasks. In stage 2, after each partner had been assigned a sub-task, each participant started to perform the information seeking process individually. Once a piece of information was found (e.g. once a sub-task was completed), the participants usually paused and notified their partner about the completion of this sub-task by discussing the outcome and search results found (Stage 3). The discussion in stage 3 always revolved around three main aspects: division of labour, making sense of the results and reviewing the remaining sub-tasks. Stages 2 and 3 were then repeated until the task is completed. However, in some cases a participant interrupted his/her partner during a task. Two main reasons were noticed for such behaviour. One reason was that the participants would need to browse search results together either to collaboratively make sense of the retrieved information or in some cases VI participants would face difficulties in viewing large volumes of search results, due to the limitations of speech-based screen-readers, and so asked their sighted partners for assistance. The other reason was that some websites were inaccessible and it was impossible for the VI partner to complete the task individually. In the observed sessions, a sum of 17 instances were recorded where the VI participants asked for assistance from their sighted partners in the co-located setting. , 13 of these cases were accessibility issues while four of them were related to navigating large result sets.

In all distributed sessions, a common pattern for the execution of the stages was observed. After the initial division of labour, both participants performed the Information seeking tasks individually and shared the results via email or instant messages. Unlike the process in the co-located session, in the distributed sessions there was no evidence that participants discussed division of labour later in the process. It was observed. However, if one participant completed all the tasks assigned to him/her, they would decide to complete their partner's outstanding tasks. Additionally, there were virtually no interactions between partners relating to making sense of retrieved results. There were only three requests for assistance recorded and all were access related.

\subsection{Stages of the Information Seeking Process}

For the most part, the separate stages of the information seeking process were done individually. Nevertheless in the co-located sessions, a number of instances were recorded in which query formulation, results exploration, query re-formulation and the search result management stages were accomplished collaboratively.

\subsubsection{Query formulation}

When a participant is assigned a particular task, he/she immediately opened a search engine and entered a query keyword. Usually the initial query would be broad and once a relevant result set is found, the participant might choose to narrow down the search to a more specific query with more keywords to obtain the information they need. However, this was not the case with VI users, as the average length of the initial query entered by sighted participants was 2.65 (SD: 0.84) in the co-located setting and 2.93 (SD: 0.54 ) in the distributed setting, while the average length of the initial query entered by the VI participants was 3.37(SD: 0.96) in the co-located setting and 3.31 (SD: 0.95) in the distributed setting. In both settings, the average length of queries by sighted participants is shorter than that of VI users. The result were statistically significant in the co-located setting at $(\mathrm{t}(26))=2.1058$, $\mathrm{p}=0.045)$ and not statistically significant in the distributed setting at $(\mathrm{t}(26)=1.2818, \mathrm{p}=0.2112)$. This result agrees with a previous comparative study [22] of the search behavior of VI and sighted users. In interviews conducted as part of [22], VI users confirmed that they often try to express their complete information need in a long, precise query, in an attempt to reduce the number of results they need to browse to reach the desired result.

Returning to the present study, a number of instances of collaboration were observed at this stage; participants sometimes suggested query keywords for his/her partner. In all co-located experiments, the average of 0.36 (SD: 0.66) instances of suggesting query terms have been recorded, while only one case was recorded in the distributed setting. In situations where the participant was unable to find results that satisfied the information need, his/her partner usually suggested another query keyword. This suggestion was either based on prior knowledge, or based on the context of the task. For instance in the conversation extract below, one participant was finding a hotel in Los Angeles (L.A). This participant suggested the query keyword for her partner, who was looking for a restaurant to dine in L.A. She suggested that the restaurant had to be near the hotel, as shown in the excerpt below:

$$
\begin{aligned}
& \text { SP: "I will look for a place to dine in L.A." } \\
& \text { VP: "You can Google restaurants near Beverly Hills" }
\end{aligned}
$$

\subsubsection{Search Result Exploration}

The number of search results explored by sighted users (average 7.14, SD: 3.37) is statistically significantly higher than that for VI participants (average 3.92, SD: 2.12) with $(\mathrm{t}(26)=2.798, \mathrm{p}=0.009)$ in the co-located setting. In the distributed setting, although the difference was smaller, it was still statistically significant $(t(26)=$ 2.32, $\mathrm{p}=0.03$ ). It was recorded that the average number of search results explored by sighted users (average 6.79, SD: 2.38) and by VI users (4.71, SD: 2.64). Collaboratively exploring a set of 
search results was commonly observed in the co-located setting only. In all sessions, an average of 3.75 (SD: 1.25) instances of exploring results collaboratively were recorded. The average number of search results viewed collaboratively is 0.5 (SD: 1.38). All such collaboratively obtained results were triggered by the VI partner needing to explore more results faster. An example of comments taken from two different studies in which the VI partner asked the sighted partner to assist when exploring the search results is shown below

From Study \#3 co-located session: VP: "Could you just glance at these results yourself?!"

From Study \#2 co-located sessions: VP: "It is listing a number of places, Can you see L.A. there?"

\subsubsection{Query Re-formulation}

This stage occurs when the user is not satisfied with the initial retrieved set of results and chooses to submit a new query. The new query might be a term from prior knowledge or from information that was just found. Most of the time this stage was done individually with an average of 1.07 (SD: 1.14) in the colocated setting and an average of 0.7 (SD: 1.24) in the distributed setting by VI participants. Re-formulation of queries by sighted participants had an average of 2.93 (SD: 2.47) in the co-located setting and an average of 1.29 (SD: 1.98) in the distributed setting. It was observed that this stage was performed collaboratively in only 3 instances in the co-located setting. In these instances the partner interrupts and suggests a query term when one partner is not satisfied with the set of results. An excerpt of a conversation that captures query re-formulation accomplished collaboratively is shown below:

From Study \#4 SP:" I think, perhaps Virgin Atlantic doesn't have direct flights to Las Vegas".

$V P$ : "Yes, this is what I was thinking about".

SP: "Let us try another keyword; perhaps you can Google direct flights to Las Vegas".

\subsubsection{Managing Search Results}

Since the task was conducted in one session, users did not employ favorites or bookmarks to keep track of required information. Sighted users tended to open multiple tabs within a browser window whereas VI users tended to open multiple windows to keep track of retrieved information. In the co-located setting, the most used note taking tool was Microsoft word. 10 VI participants and 7 sighted participants have used it. The next most used tool by sighted participants was using pen and paper; four sighted users requested a pen and paper to note down retrieved information. Additionally two VI participants and one sighted user used the Microsoft note pad application. One participant established skype communication and added the retrieved information into the Skype session. In most of the conducted sessions, both participants would store the retrieved information in one of the previously mentioned forms. However, in three sessions only one participant noted down the retrieved information and the other participants entirely depended on their partners. In two sessions it was the sighted users who kept track of the retrieved information and stored it, while in one session it was the VI user who organized and stored the retrieved information in a word file.

In the distributed setting, the most used note taking tool was Microsoft word with nine VI participants and five sighted participants. Three sighted participants requested pen and paper to take notes, and two VI participants used the Microsoft notepad application. In the distributed setting, three VI participants and four sighted participants preferred storing their notes and retrieved information using the communication tool, which was either email or chat messaging. In these situations, one team member usually kept track of the information shared in the communication tool by storing them in a word processing application. Four VI participants and three sighted participants kept track of the retrieved in information received from their partners and stored them in a Microsoft word file.

It was observed that the information noted down or exchanged by participants was of five types. These were: a website link, a website link with details, details about the sub-task, simply keywords that refer to the information or copying a part of the webpage. Figure 1 shows the percentage of each identified category in the co-located and the distributed settings.

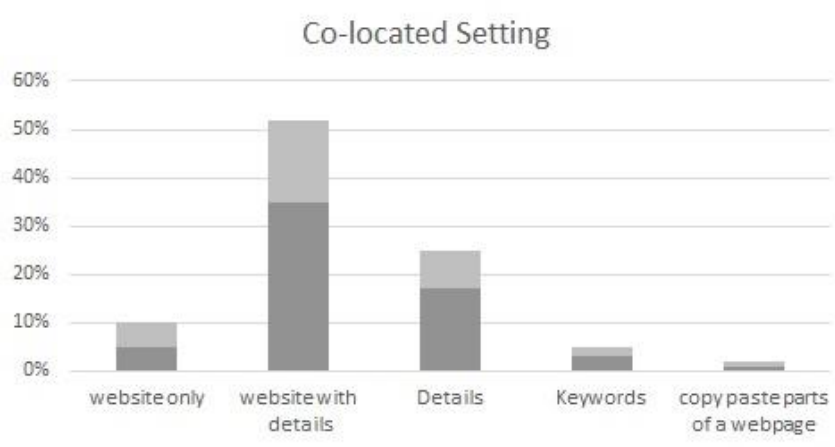

Distributed Setting

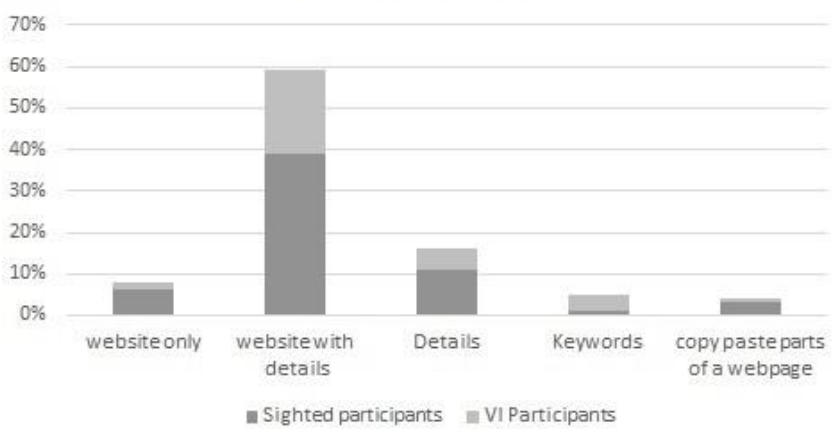

Figure 1. The total percentage occurrence of each type of information kept or exchanged in both co-located and distributed sessions

As shown in Figure 1, the majority of information kept by both sighted and VI users in both settings were either website links with details $(52 \%$ in the co-located setting and $59 \%$ in the distributed setting) or details only (25\% in the co-located setting and $16 \%$ in the distributed setting). Moreover, the amount of information kept by VI users is nearly half the amount of information kept by sighted users. In fact in the distributed setting, sighted users exchange rate of information to VI users was $2: 1$. In the post-study interviews, seven VI participants highlighted the difficulties of having to switch between three different applications: the web browser, the email client and the note taking tool during the process

In the co-located setting the retrieved information was noted down but was not exchanged between the participants by any means. The participants were merely verbally notifying their partners about their progress or asking about their partner's progress as a means of updating their awareness information. Whilst in the 
distributed setting, partners exchanged information by email or instant messaging as well as using note taking tool.

\subsection{Time intervals}

We observe the time spent by participants on each stage. This includes time spent entering a query, times spent viewing search results pages, time spent browsing websites, and time spent managing information. In addition, time spent dealing with an error (whether it's a connection error, interface error or accessibility issue) and time spent switching from one application to another. Table 2 shows the average time interval spent in each stage in both settings. The figures shows that the most apparent differences between the two groups of users were in the results exploration stage, retrieved information management stage, communication stage and switching from one application to another. In the results exploration stage in both settings VI users spent on average a longer time then their sighted partners. Though the differences were not statistically significant with t-test results ( $\mathrm{t}(13)=2.05, \mathrm{p}=0.06)$ in the distributed setting and $(\mathrm{t}(13)=1.95$, $\mathrm{p}=0.7)$ in the co-located setting. According to our observations the main reason that made the VI participants spend on average more time in this stage is the serial nature of speech that would make the process of going through search results longer.

\begin{tabular}{|l|l|l|l|l|}
\hline & \multicolumn{2}{|c|}{ Co-located } & \multicolumn{2}{c|}{ Distributed } \\
\hline & $\begin{array}{c}\text { VI } \\
\text { participant }\end{array}$ & $\begin{array}{c}\text { Sighted } \\
\text { participant }\end{array}$ & $\begin{array}{c}\text { VI } \\
\text { Participant }\end{array}$ & $\begin{array}{c}\text { Sighted } \\
\text { Participant }\end{array}$ \\
\hline $\begin{array}{l}\text { Entering } \\
\text { query term }\end{array}$ & 2.38 & 2.08 & 2.51 & 1.37 \\
{$[1: 12]$} & {$[1: 59]$} & {$[2: 01]$} & {$[0: 58]$} \\
\hline $\begin{array}{l}\text { Exploring } \\
\text { search results }\end{array}$ & $3: 58$ & $2: 11$ & $3: 17$ & $2: 07$ \\
\hline Browsing & $12: 39]$ & {$[1: 49]$} & {$[1: 52]$} & {$[1: 17]$} \\
websites & {$[8: 48]$} & {$[8: 47]$} & $10: 44$ & $11: 49$ \\
\hline $\begin{array}{l}\text { Managing } \\
\text { information }\end{array}$ & $2: 50$ & $5: 57$ & $1: 59$ & {$[6: 08]$} \\
\hline Chat & $0: 00$ & {$[3: 06]$} & {$[1: 52]$} & $2: 50$ \\
& & $0: 00$ & $06: 56$ & $83: 52]$ \\
\hline Encountering & $0: 23$ & $0: 00$ & $0: 20$ & {$[4: 37]$} \\
error & {$[0: 43]$} & & {$[0: 28]$} & $0: 01$ \\
\hline Switching & $1: 45$ & $0: 35$ & $1: 21$ & $0: 02]$ \\
applications & {$[0: 19]$} & {$[0: 31]$} & {$[0: 34]$} & {$[0: 27]$} \\
\hline
\end{tabular}

Table 2. The average time interval spent (in seconds) in each stage in both settings by both groups of users in the study (Mean [standard deviation])

In managing retrieved information, sighted participants spent longer time in both settings. However, the differences are not statistically significant using at $(t(13)=1.95, p=0.72)$ in the colocated setting at $(\mathrm{t}(13)=2.05, \mathrm{p}=0.06)$ in the distributed setting. Additionally, it was observed that VI users spent considerably more time switching from one application to another. The applications were internet explorer, the email client or instant chat application in the distributed setting and word processing document or note pad. In the post-study interviews eight VI participants have highlighted the difficulties of having to switch between the three different applications.

The average time consumed browsing web search results by both groups was significantly higher in the co-located setting with t-test results $(\mathrm{t}(26)=2.27, \mathrm{p}=0.03)$. As in the distributed setting participants spent a considerable about of time using the email client or instant chat messaging service to provide their partners with updates about their progress.

\section{FINDINGS AND DISCUSSION}

\subsection{CIS Process}

Clearly identifying the stages of the CIS process was not among the formulated research questions; however having an insight of the stages would be of benefit and can help in identifying the phases which are influenced by the presence of two different modalities. According to the literature, the process of CIS is not well-defined and can largely differ according to the task performed. London [13] introduced a general model of collaborative activity. The model comprises three main phases: (1) the problem setting phase in which collaborators spend time understanding the problem and identifying resources required for solving it. (2) A direction setting phase which involves organizing group activities and agreeing on actions, and (3) the implementation phase in which collaborators complete the task assigned to them. He emphasize that this stage can differ according to application area and group size.

We observed a similar structure in the current study. The pairs started by discussing and making sense of the given task. They then assigned different subtasks to each other and started conducting the information seeking task individually. As seen in the analysis section, it was observed that in cases when the partners were colleagues, the VI partner would delegate the task that might contain an inaccessible interaction to the sighted partner. This action contributed to enhancing the performance of the pairs and their efficiency in completing the task.

There was clear evidence of similarities between the stages of the individual IS process and stages of collaborative IS as reported by Hyldegard [8] and Shah and Gonzalez-Ibanez [26]. Even though participants often performed the IS task individually before sharing the results with their partners, as shown in the analysis section, some stages were performed collaboratively for various reasons. The frequency of collaboration largely differed; it occurred mostly in the search results exploration stage in the colocated sessions and in the results management stages in both settings.

\subsection{Result Exploration}

Collaboration was triggered when the VI participant would ask the sighted participant to assist in going through a large volume of search results. Examination of large sets of search results by Speech-based screen-readers can be time consuming and imposes a number of challenges such as short term memory overload and a lack of contextual information about the data presented [19][22][28]. Additionally, current screen-readers provide almost no mechanism for overviewing a set of search results.

\subsection{Results Management}

The results management stage was also done collaboratively. The motivation behind users' collaboration in this stage was that they were encouraged to collaboratively work together and produce one outcome at the end of the task. In three of the co-located experiments, only one team member was taking notes, while in seven of the distributed studies only one team member was taking notes. 
The observations showed that the amount of information kept and noted down by sighted participants was higher than the amount of retrieved information kept and exchange by VI participants. This is likely to be the result of two factors. The first being that sighted users viewed more results than VI users and hence they kept and exchanged more retrieved information. The second factor is related to the cognitive overhead and time delays that VI users encounter when switching between the web browser and an external application used to take notes. This itself is likely to increase the cognitive load on VI users and hence slow down the process. The effect of this factor was more apparent in the distributed setting where VI users were required to switch between three applications: the email client or instant chat application, the web browser and note taking application. The amount of information kept and exchanged by sighted users was more than double the information kept and exchanged by VI participants reported in the analysis section.

\section{IMPLICATIONS AND FUTURE WORK}

The results and findings of our study clearly indicate that there are a number of ways that the CCIS process could be made more accessible and that the tools used currently do not address the process adequately. The motivation to improve this situation is strengthened by the frequency of team working both in education and employment [15], of which web searching often forms an important part. This section discusses the implications of the current study for the design of technical solutions to support CIS in a cross-modal context, some of which will form future work in this project.

\subsection{Providing an Overview of Search Results}

The search results exploration was a stage that was done collaboratively in many instances. The reason behind this sort of collaboration is that the VI participants needed help from their sighted partners to navigate through a large volume of search results. This also was highlighted by studies that compared VI individual information seeking behaviour with sighted information seeking behavior [9][22]. These studies have stressed that this stage is the most challenging and time consuming stage for VI users. In fact, this stage is assessed to be two times longer when performed by VI users than sighted users [9]. The sequential nature of speech rendered by screen-readers over loads the user's short-term memory and provides no means of representing spatial layout and contextual information of web pages [19]. Developing a mechanism that provides VI group members with an overview of search results and the ability to zoom in on particular information of interest could help in increasing the VI group members' independence in CCIS activities.

\subsection{Improving the Management and Sharing of Search Results}

It was observed in the study that managing search results was one of the main obstacles faced by VI users. This was more apparent in the distributed setting where the user is required to switch between three different applications. Moreover, observations in both settings highlighted differences in individual approaches employed by sighted and VI users when managing search results.

A recent publication by Sahib et al. [23] described an integrated tool that allows VI users to keep track of their search progress, and manage their search results. An evaluation of the tool with VI participants resulted in high satisfaction rates as they found it easier to handle search results within the tool as it removed the overhead of switching between a number of applications. Extending this approach to a collaborative context, including tracking the search progress of all collaborating searchers and the management of results at the individual and group levels, appears to be worth investigating, to see if similar or additional advantages accrue. However, a utility that allows collaborators to recall the websites visited and query keywords entered by their partners is clearly not sufficient, as our findings showed the majority of information exchanged regarding search results included website links and details of the information retrieved. Therefore, a tool to support CCIS needs to provide better integration of the whole process as well as supporting the sharing of websites and details of results found. An additional complexity in providing an integrated tool in the context of CIS, as opposed to individual IS, is that this tool will be shared between users employing different interaction modes, and must be accessible through these different modes.

A tool like SearchTeam ${ }^{2}$, which is a commercially available website for collaborative search, provides the collaborators with a common place to share details of websites, links and comments. This is similar to a feature that Diigo [10], a collaborative bookmarking website, provides that allows users to share bookmarks.

\subsection{Improving Awareness of the Search Query Terms and Search Results}

Allowing collaborators to know the query terms entered and results viewed by their partners would certainly inform them about their partner's progress toward the completion of a task. Additionally, having a view of partners search results can allow sighted users to collaborate with their VI partners while going through large amounts of search results. A tool like Coagmento [24] provides a mechanism to allow users to be aware of their team members' activities. This is done by dedicated views that are updated with query terms used and web pages viewed by collaborators. Co-sense is a Microsoft developed CIS system that is aimed to support sensemaking in collaborative web search tasks. It provides different views of the collaborators' activities in terms of query keywords; documents retrieved and shared comments [20]. In the CCIS context, features and views provided by interfaces such as Coagmento and Co-Sense, could be tailored to be screen-reader friendly, by supporting rapid navigation to different views using keystrokes and providing keyword summaries of activities. The WeSearch system [17] allows sharing queries and comments within the group. The queries and comments are colour coded by collaborators. This could be implemented within the context of CCIS by using different screenreader voices and/or spatially distributing the auditory representations of queries and comments made by different group members.

\section{CONCLUSIONS}

This paper describes an exploratory study that examines the effect of cross-modal collaboration on the stages of information seeking in co-located and distributed settings. The findings show that there is a clear influence of the different modalities and settings on the

\footnotetext{
${ }^{2}$ http://searchteam.com/
} 
different stages of information seeking. The most apparent collaborative issues occurred in the results exploration and management stages. Some of these problems have an underlying accessibility issue caused by to the limitations in the way information is presented and navigated using speech-based screen readers. This paper concludes by proposing some design implications that can be considered when designing a tool that supports CCIS. The results of the study highlight that considerably more work needs to be done to improve the accessibility of both individual and cross-modal information seeking.

\section{REFERENCES}

[1] Al-Thani, D., Stockman, T., Tombros, A., (2013) Cross-Modal Collaborative Information Seeking (CCIS): An Exploratory Study. In Proceeding of the 27th International British Computer Society Human Computer Interaction Conference. London, UK .

[2] Andronico, P., Buzzi, M., Castillo, C., \& Leporini, B. (2006). Improving search engine interfaces for blind users: a case study. Universal Access in the Information Society.

[3] Caldwell, B. (2008). Web content accessibility guidelines (WCAG) 2.0. W3C.

[4] Corbin, J. M., \& Strauss, A. C. (2008). Basics of Qualitative Research: Techniques and Procedures for Developing Grounded Theory (Third ed.). Sage Publications, Inc.

[5] Craven, J., Brophy, P., \& Britain, G. (2003). Non-visual Access to the Digital Library: The Use of the Digital Library Interfaces by Blind and Visually Impaired People. Manchester Metropolitan University, Centre for Research in Library and Information Management.

[6] Golovchinsky, G., Pickens, J., and Back, M. A taxonomy of collaboration in online information seeking. In JCDL Workshop on Collaborative Information Retrieval, 2008.

[7] Harper, S., \& Yesilada, Y. (2008). Web accessibility: a foundation for research. Springer.

[8] Hyldegard ,J. (2009). Beyond the search process-Exploring group members' information behavior in context. Information Processing \& Management, 45(1), 142-158.

[9] Ivory, M. Y., Yu, S., \& Gronemyer, K. (2004). Search result exploration: a preliminary study of blind and sighted users' decision making and performance. In Proceeding CHI'04 extended abstracts on Human factors in computing systems.

[10] Kelly, R. M., \& Payne, S. J., 2014. Collaborative web search in context: A study of tool use in everyday tasks. In Proceedings of ACM conference on Computer-Supported Cooperative Work.

[11] Kuhlthau, C. C. (1991). Inside the search process: Information seeking from the user's perspective. Journal of the American Society for Information Science.

[12] Large, A., Beheshti, J., \& Rahman, T. (2002). Gender differences in collaborative Web searching behavior: an elementary school study. Information Processing \& Management, 38(3), 427-443.

[13] London, S. (1995). Collaboration and community. Richmond, VA, Pew Partnership for Civic Change, University of Richmond.
[14] Marchionini, G., \& White, R. (2008). Find what you need, understand what you find. International Journal of Human Computer Interaction, 23(3), 205-237.

[15] Morris, M. R. (2008). A survey of collaborative web search practices. Proceedings of the twenty-sixth annual SIGCHI conference on Human factors in computing systems, In Proceeding CHI '08 (pp. 1657-1660). New York, NY, USA.

[16] Morris, M. R. (2013). Collaborative search revisited. Proceedings of the 2013 conference on Computer supported cooperative work (pp. 1181-1192). New York, NY, USA.

[17] Morris, M. R., \& Horvitz, E. (2007). SearchTogether: an interface for collaborative web search. In Proceeding of the 20th annual ACM symposium on User interface software and technology (pp. 3-12).

[18] Morris, M. R., Lombardo, J., \& Wigdor, D. (2010). WeSearch: supporting collaborative search and sensemaking on a tabletop display. In Proceeding of the 2010 ACM conference on Computer supported cooperative work.

[19] Murphy, E., Kuber, R., Strain, P., McAllister, G., \& Yu, W. (2007). Developing sounds for a multimodal interface: conveying spatial information to visually impaired web users. In Proceeding of the International Conference on Auditory Display (pp. 26-29).

[20] Pickens, J., Golovchinsky, G., Shah, C., Qvarfordt, P., \& Back, M. (2008). Algorithmic mediation for collaborative exploratory search. In Proceeding of the 31 st annual international ACM SIGIR conference on Research and development in information retrieval, SIGIR '08 (pp. 315322). New York, NY, USA.

[21] Paul, S. A., \& Morris, M. R. (2009). CoSense: enhancing sensemaking for collaborative web search. In Proceeding of the 27th international conference on Human factors in computing systems, CHI '09. New York, NY, USA.

[22] Sahib, N. G., Tombros, A., \& Stockman, T. (2012) A comparative analysis of the information seeking behavior of visually impaired and sighted searchers. Journal of the American Society for Information Science and Technology.

[23] Sahib, N., Tombros, A., Stockman, T. (2013) Evaluating a search interface for visually impaired users. Submission to the Journal of the American Society for Information Science and Technology.

[24] Shah, C. (2009). Toward Collaborative Information Seeking (CIS). In Proceeding of 1 st collaborative information retrieval workshop, JCDL.

[25] Shah, C. (2010). Coagmento-a collaborative information seeking, synthesis and sense-making framework. Integrated demo at CSCW, 6-11.

[26] Shah, C., \& González-Ibáñez, R. (2010). Exploring information seeking processes in collaborative search tasks. In Proceeding of the American Society for Information Science and Technology, 47(1), 1-7.

[27] Shah, C., \& Marchionini, G. (2010). Awareness in collaborative information seeking. Journal of the American Society for Information Science and Technology, 61(10), 1970-1986.

[28] Stockman, T., \& Metatla, O. (2008). The influence of screenreaders on web cognition. In Proceeding of Accessible design in the digital world conference (ADDW 2008), York, UK. 